\title{
Institution of localized high-frequency electrical stimulation targeting early myocardial infarction: Effects on left ventricle function and geometry
}

\author{
Michael C. Genau, BS, ${ }^{\text {a }}$ Paige E. Perreault, BS, ${ }^{a}$ Eva Romito, PhD, ${ }^{a}$ Heather Doviak, BS, ${ }^{a}$ \\ Christina B. Logdon, BS, ${ }^{a}$ Stephen Ruble, PhD, ${ }^{b}$ and Francis G. Spinale, MD, PhD ${ }^{\mathrm{a}, \mathrm{c}}$
}

\begin{abstract}
Background: Although strategies have focused on myocardial salvage/regeneration in the context of an acute coronary syndrome and a myocardial infarction (MI), interventions targeting the formed MI region and altering the course of the post-MI remodeling process have not been as well studied. This study tested the hypothesis that localized high-frequency stimulation instituted within a formed MI region using low-amplitude electrical pulses would favorably change the trajectory of changes in left ventricle geometry and function.
\end{abstract}

Methods: At 7 days following MI induction, pigs were randomized for localized high-frequency stimulation ( $\mathrm{n}=5,240 \mathrm{bpm}, 0.8 \mathrm{~V}$, and $0.05 \mathrm{~ms}$ pulses) or unstimulated $(n=6)$. Left ventricle geometry and function were measured at baseline (pre-MI) and at 7, 14, 21, and 28 days post-MI using echocardiography. MI size at 28 days post-MI was determined by histochemical staining and planimetry.

Results: At 7 days post-MI and before randomization to localized high-frequency stimulation, left ventricular ejection fraction and end-diastolic volume was equivalent. However, when compared with 7-day post-MI values, left ventricle enddiastolic volume increased in a time-dependent manner in the MI unstimulated group, but the relative increase in left ventricle end-diastolic volume was reduced in the MI localized high-frequency stimulation group. For example, by 28 days post-MI, left ventricle end-diastolic volume increased by $32 \%$ in the MI unstimulated group but only by $12 \%$ in the MI localized high-frequency stimulation group $(P<.05)$. Whereas left ventricular ejection fraction appeared unchanged between MI groups, estimates of pulmonary capillary wedge pressure, a marker of adverse left ventricle performance and progression to failure, increased by $62 \%$ in the MI unstimulated group and actually decreased by $17 \%$ in the MI localized highfrequency stimulation group when compared with 7-day post-MI values $(P<.05)$. MI size was equivalent between the MI groups, indicative of no difference in the extent of absolute myocardial injury.

Conclusions: The unique findings from this study are 2-fold. First, targeting the MI region following the resolution of the acute event using a localized stimulation approach is feasible. Second, localized stimulation modified a key parameter of adverse post-MI remodeling (dilation) and progression to heart failure. These findings demonstrate that the MI region itself is a modifiable tissue and responsive to localized electrical stimulation. (J Thorac Cardiovasc Surg 2018;156:568-75)

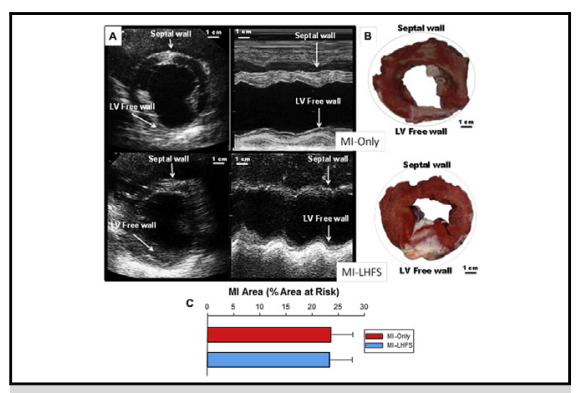

Left ventricle cross-sectional and targeted echocardiographic views shown with concordant short-axis.

\section{Central Message}

The findings of this study demonstrate that the MI region itself is modifiable tissue and responsive to localized electrical stimulation.

\section{Perspective}

This project utilized a localized highfrequency, low-amplitude stimulus contained within the MI region over a 21-day period in a large animal model. This approach favorably modified adverse post-MI tissue, demonstrating that this region is a modifiable tissue and responsive to localized electrical stimulation.

See Editorial Commentary page 576.

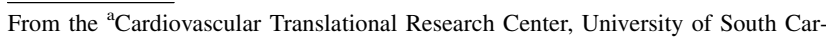
olina School of Medicine, Columbia, SC; ${ }^{b}$ Boston Scientific Corporation, Minneapolis, Minn; and ${ }^{\mathrm{c} W i l l i a m ~ J e n n i n g s ~ B r y a n ~ D o r n ~ V A ~ M e d i c a l ~ C e n t e r, ~ C o l u m b i a, ~}$ SC.

Supported by a National Institute of Health Small business Technology Grant (No. 1R42HL112519) and a Merit Award from the Veterans' Affairs Health Administration.

Received for publication May 3, 2017; revisions received Dec 20, 2017; accepted for publication Jan 13, 2018; available ahead of print March 30, 2018.

\footnotetext{
Address for reprints: Francis G. Spinale, MD, PhD, Cardiovascular Translational Research Center, University of South Carolina School of Medicine, 6439 Garners Ferry Rd, Columbia, SC 29208 (E-mail: cvctrc@uscmed.sc.edu). 0022-5223/\$36.00

Copyright (C) 2018 Published by Elsevier Inc. on behalf of The American Association for Thoracic Surgery

https://doi.org/10.1016/j.jtcvs.2018.01.104
} 

Abbreviations and Acronyms
LHFS = localized high frequency stimulation
$\mathrm{LV} \quad=$ left ventricle
ANOVA $=$ analysis of variance
MI = myocardial infarction
PCWP = pulmonary capillary wedge pressure

Scanning this QR code will take you

to the article title page.

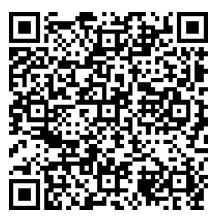

Cardiovascular disease, in which ischemic heart disease and myocardial infarction (MI) constitute a significant proportion of this disease category, is a leading cause of morbidity and mortality. Although there have been significant advances with respect to the use of thrombolytic agents and myocardial salvage at the time of an acute coronary syndrome, long-term prognoses remain poor for a number of patients who have residual myocardial injury coalescing into an MI. For example, in the Survival and Ventricular Enlargement trial, more than one-third of patients who had received optimal reperfusion had poor outcomes, such as adverse post-MI remodeling and heart failure. ${ }^{1}$ Thus, despite optimal initial therapeutic intervention and standard pharmacologic care, long-term prognosis for a large number of post-MI patients remains poor. Although significant basic and translational focus has been on understanding the pathways and developing treatment strategies to reduce the initial injury and magnitude of the MI, advancements in therapeutic strategies that target the residual, maturing infarct scar have been incremental. One reason for this disparity in research and development focus has likely been due to the previously held canonical belief that the resultant MI is a nonmodifiable tissue. However, it is now recognized that the MI region-even weeks to months post-MI-is a dynamic tissue structure containing a population of transdifferentiated fibroblasts, continuous extracellular matrix synthesis and proteolysis, and dynamic remodeling (ie, infarct expansion). ${ }^{2-10}$ Thus, the development of strategies that specifically target the formed MI and thereby attenuate/reverse the progression of adverse left ventricle (LV) remodeling in patients with a pre-existing MI not only constitutes an important and growing unmet medical need but also likely holds significant therapeutic potential. In a past proof-of-concept study, the MI region was successfully subjected to continuous, localized high-frequency stimulation (LHFS). ${ }^{11}$ However, that past study did not examine whether LHFS could be instituted in a more clinically relevant time point post-MI and whether and to what degree LHFS would modify the natural history of post-MI remodeling. Accordingly, the overall goal of this study was to examine the effects of LHFS in a large animal model of post-MI remodeling in terms of LV geometry and function. The central hypothesis to be tested was that when LHFS is instituted following the resolution of the acute MI period (ie, 7 days post-MI), the progression of adverse post-MI remodeling, specifically defined as LV dilation, would be attenuated.

\section{METHODS AND RATIONALE}

The goal of these studies was to examine whether and to what degree the institution of LHFS early post-MI resulted in a modifiable and favorable effect on the natural history of post-MI remodeling. In our previous studies, we have established that a high-frequency, low-amplitude electrical stimulus directly delivered to the central portion of the MI region did not result in propagation of the electrical impulse beyond the MI region and was not associated with susceptibility to arrhythmias. ${ }^{11}$ Accordingly, the present study used this LHFS stimulation algorithm and instituted LHFS at 7 days following MI induction. LV function and geometry were serially quantified by echocardiography. ${ }^{6,12}$ The rationale for selecting the 7-day post-MI time point for the institution of LHFS was predicated upon several considerations. First, the intent of this study was not to modify the initial MI injury, but rather move past the initial wound healing response and determine whether the natural history of the MI remodeling process could be modified. Second, cardiovascular procedures, such as coronary revascularization, have been shown to be feasible and are at less risk at approximately 5 to 7 days following the acute coronary syndrome. ${ }^{13-15}$ Third, the delay of instituting LHFS until an early post-MI time point holds translational significance in that this would be a likely time interval in which clinical judgment about the size and probable scope of the MI can be determined and thus consider patients for additional interventional therapies, such as LHFS.

With the above goals in mind and based on previous studies, the primary response variable for the current study was that which fundamentally defines adverse post-MI remodeling: LV dilation. For the purposes of this study, which was specifically defined as LV end-diastolic volume because past studies have identified this to be an important index for clinical outcomes and progression to heart failure..$^{1-4,16-18}$ Based on our past studies using pharmacologic approaches to attenuate post-MI remodeling and our initial study using LHFS, ${ }^{6,11,12}$ we set the criteria that a relative reduction of approximately $30 \%$ in LV dilation from untreated post-MI values must occur to be considered a relevant positive response. This relative reduction in LV volume has been used as a set point for clinical trials previously. ${ }^{16-18}$ Secondary response variables included changes in LV function and wall thickness during the post-MI period.

\section{Induction}

Permanent coronary ligation was performed in mature Yorkshire pigs (Hambone Farms, Orangeburg, SC) $(\mathrm{n}=11,25 \mathrm{~kg})$ as previously described. ${ }^{6,11,12}$ Briefly, the pigs were anesthetized (2.5\% isoflurane) and the $\mathrm{LV}$ was accessed through a small $(3 \mathrm{~cm})$ left-sided thoracotomy. MI was induced by direct ligation of the first 2 obtuse marginal arteries (obtuse marginal 1 and obtuse marginal 2) at the origin from the circumflex coronary artery (4.0 silk suture), which we have established previously results in a consistent MI size ( $25 \%$ of area at risk). ${ }^{12}$ At the midregion between obtuse marginal 1 and obtuse marginal 2, a shielded stimulating electrode (Myopore Biopolar Sutureless Pacing Lead; Greatbatch Medical, Frisco, Tex) was placed into the midmyocardium of the MI. The pacing lead was then connected to a modified pacemaker (Boston Scientific, 
Marlborough, Mass) that allowed for high-rate pacing. The pacemaker was placed in a subcutaneous pocket and the incision closed. All animals were treated and cared for in accordance with the National Institutes of Health Guide for the Care and Use of Laboratory Animals, Eighth Edition, ${ }^{19}$ and all protocols were approved by the University of South Carolina's Institutional Animal Care and Use Committee.

\section{Randomization and LHFS Protocol}

At 24 hours post-MI induction, the pigs were randomly assigned to undergo LHFS or for the pacemaker to remain deactivated for the entire postMI protocol. Randomization was performed in an alternating fashion with each animal assigned a code number, which was not broken until the completion of the full protocol.

The LHFS protocol was instituted at 7 days post-MI and the stimulation parameters, which had been validated previously, ${ }^{11}$ were a frequency of $240 \mathrm{bpm}$, an amplitude of $0.8 \mathrm{~V}$, and pulse duration of $0.05 \mathrm{~ms}$. With the exception of the echocardiography studies as described in the following paragraph, LHFS was maintained continuously from post-MI day 7 through post-MI day 28 and thus constituted a 21 day stimulation period. Confirmation that the LHFS stimulation parameters were operative throughout this period of time was performed through regular (intervals of 3-5 days) pacemaker interrogation (3120 Programmer, Boston Scientific).

\section{Echocardiography}

The day before randomization and MI induction, the animals were sedated (diazepam, $200 \mathrm{mg}$ orally) and echocardiography performed (Vivid 7 Dimension Ultrasound System M4S 1.5-4.3 MHz active matrix array sector transducer probe; GE Healthcare, Chicago, Ill) to measure LV volumes, posterior LV free-wall thickness, ejection fraction, and estimates of pulmonary capillary wedge pressure (PCWP) as described previously. ${ }^{11,12}$ Briefly, LV volumes were determined using the method-ofdisks variant of Simpson's algorithm mitral valve inflow velocities, and tissue Doppler was used to compute an estimate of PCWP. ${ }^{12,20,21}$ The pigs were returned to the laboratory under identical sedation/study conditions at 7, 14, 21, and 28 days post-MI. In this study, all echocardiographic measurements were performed by a single observer (CBL) who was blinded to the group assignment until the completion of the study. Past clinical studies have identified that using Doppler echocardiography provides a reasonable estimate of PCWP and LV filling pressures, particularly in the operative ranges of LV function anticipated in the present study. ${ }^{20,21}$

\section{Terminal Studies and MI Size Computations}

At the conclusion of the day 28 post-MI time point and under full surgical anesthesia (5\% isoflurane), the LV was harvested, and following confirmation of lead placement at the central portion of the MI region, a full circumferential section $(5 \mathrm{~mm})$ of the LV was taken at $2 \mathrm{~cm}$ below the obtuse marginal 1 and obtuse marginal 2 ligation sutures. The LV sections were incubated in triphenyl tetrazolium chloride solution (room temperature, 20 minutes) and then digital images of these stained sections were subjected to densitometry. The infarct size was determined by convention, whereby the area negative for triphenyl tetrazolium chloride staining (infarct) was normalized to the total area at risk (circumflex artery distribution area) and expressed as a percent. ${ }^{12}$

\section{Computation and Data Analysis}

Statistical analyses were performed using STATA statistical software (STATA Corp, College Station, Tex). LV geometry, function, and area within the markers were compared between the control and MI groups using a 1-way analysis of variance (ANOVA). A multiway ANOVA was performed, evaluating for treatment dependent and MI-dependent effects. Post hoc separation following ANOVA was performed initially with a Bonferroni analysis (prcomp module in STATA), following which changes from
post-MI 7-day values were computed and expressed as a percent change from these values. Comparisons across groups and times were performed using ANOVA and Bonferroni analysis. In addition, changes from individual values were tested against post-MI 7-day values using a $t$ test where the null hypothesis was a percent change of zero. Results are presented as a mean \pm standard error of the mean. In our past reports, we identified that pre-MI and post-MI LV end-diastolic volume, which is the primary response variable for the present study, approximated a normal distribution. ${ }^{6,11,12}$ Due to a high uniformity in animal size and LV geometry that this laboratory has reported previously, ${ }^{6,12}$ the coefficient of variation for the primary and secondary response variables was anticipated to be $<20 \%$. For the purposes of an initial power estimate for the primary response variable (LV end-diastolic volume), the degree of LV dilation and variability associated with this response variable, as reported previously in this post-MI model, was used. ${ }^{6,12}$ Based on the hypothesis that LHFS will yield a $30 \%$ reduction in the magnitude of LV dilation, a sample size of 6 would yield an $80 \%$ power (assuming an alpha level set at 0.05$)$.

\section{RESULTS}

The summary of results for this study in terms of LV function and geometry and the effects of LHFS are presented in Table 1. There was no attrition in either group following the initial MI surgery, randomization, or postMI follow-up period. The composite LV baseline (preMI) values were consistent with those reported previously. ${ }^{6,11,12}$ It should be emphasized that the LHFS randomization did not occur until after these baseline measurements and thus pooled values are provided in Table 1. However, retrospective analysis of the baseline data revealed equivalent values between the pigs randomized to the post-MI only group and the MI-LHFS group with respect to the primary response variable, LV enddiastolic volume $(39.5 \pm 0.1$ vs $41.4 \pm 0.1 \mathrm{~mL}$, respectively). The actual coefficient of variation for baseline LV end-diastolic volume was $\sim 7 \%$ and for the secondary response variables was $<18 \%$, and therefore were within anticipated ranges and consistent with past studies. ${ }^{11,12}$ The absolute values for LV geometry and function following randomization to the post-MI only group and the MI-LHFS group at 7 days post-MI are shown in Table 1. It should be emphasized that at this post-MI time point, LHFS had not been instituted, and as such, these values reflect LV function and geometry before treatment initiation. In those pigs randomized to undergo LHFS, LV end-diastolic volume was higher than those assigned to the MI-only group. At later time points, LV end-diastolic volume increased in both groups but was attenuated in the MI-LHFS group at both 21 and 28 days post-MI. A time-dependent fall in LV ejection fraction and posterior wall thickness occurred in both groups, with the decline in $\mathrm{LV}$ posterior wall thickness attenuated in the MI-LHFS group by 28 days post-MI. PCWP, an estimate of LV filling pressures, increased in a timedependent manner in both groups post-MI but was lower in the MI-LHFS group at 28 days post-MI. 
TABLE 1. Left ventricle (LV) function and geometry after myocardial infarction (MI): Effects of localized high-frequency stimulation (LHFS)

\begin{tabular}{lcccccc}
\hline \multicolumn{1}{c}{ Treatment } & Day & EF $(\%)$ & LVEDV $(\mathbf{m L})$ & LVPWThd (cm) & LVPWThs (cm) & PCWP (mm Hg) \\
\hline Baseline $(\mathrm{n}=11)$ & 0 & $59 \pm 1$ & $40.0 \pm 0.8$ & $0.74 \pm 0.01$ & $1.02 \pm 0.02$ & $4.8 \pm 0.2$ \\
MI only $(\mathrm{n}=6)$ & $7^{*}$ & $47 \pm 3 \dagger$ & $60.6 \pm 2.9 \dagger$ & $0.65 \pm 0.02$ & $0.67 \pm 0.02 \dagger$ & $6.4 \pm 0.9$ \\
& 14 & $46 \pm 1 \dagger$ & $67.9 \pm 2.0 \dagger$ & $0.60 \pm 0.02 \dagger$ & $0.63 \pm 0.02 \dagger$ & $8.1 \pm 0.6 \dagger$ \\
& 21 & $43 \pm 1 \dagger$ & $71.9 \pm 2.7 \dagger, \ddagger$ & $0.56 \pm 0.03 \dagger, \dagger$ & $0.58 \pm 0.03 \dagger, \ddagger$ & $8.4 \pm 0.8 \dagger$ \\
& 28 & $39 \pm 1 *, \dagger$ & $79.5 \pm 3.9 \dagger, \ddagger$ & $0.54 \pm 0.03 \dagger, \dagger$ & $0.56 \pm 0.02 \dagger, \ddagger$ & $9.7 \pm 0.7 \dagger, \ddagger$ \\
MI-LHFS (n=5) & $7 *$ & $47 \pm 4 \dagger$ & $66.5 \pm 3.4 \dagger$ & $0.65 \pm 0.04$ & $0.68 \pm 0.04 \dagger$ & $8.6 \pm 0.2 \dagger$ \\
& 14 & $42 \pm 4 \dagger$ & $70.6 \pm 4.1 \dagger$ & $0.63 \pm 0.04$ & $0.67 \pm 0.05 \dagger$ & $8.5 \pm 0.4 \dagger$
\end{tabular}

Values are presented as mean \pm standard deviation. $E F$, Ejection fraction; $L V E D V$, left ventricular end diastolic volume; $L V P W T h d$, diastolic left ventricular posterior wall thickness; $L V P W T h s$, systolic left ventricular posterior wall thickness; $P C W P$, pulmonary capillary wedge pressure; $M I$, myocardial infarction; LHFS, localized high frequency stimulation. *Values obtained and computed before activation of the LHFS protocol. $\dagger P<.05$ versus baseline. $\ddagger P<.05$ versus respective day 7 . $\S P<.05$ versus respective MI-only value.

LHFS was instituted at 7 days post-MI, and the indices of LV function and geometry were next computed as a function of these pretreatment, 7-day post-MI values (Figure 1). The trajectory for LV dilation and posterior wall thinning were significantly attenuated in the MILHFS group. For example, LV end-diastolic volume increased by more than $30 \%$ in the MI-only group and by approximately $10 \%$ in the MI-LHFS group. The magnitude
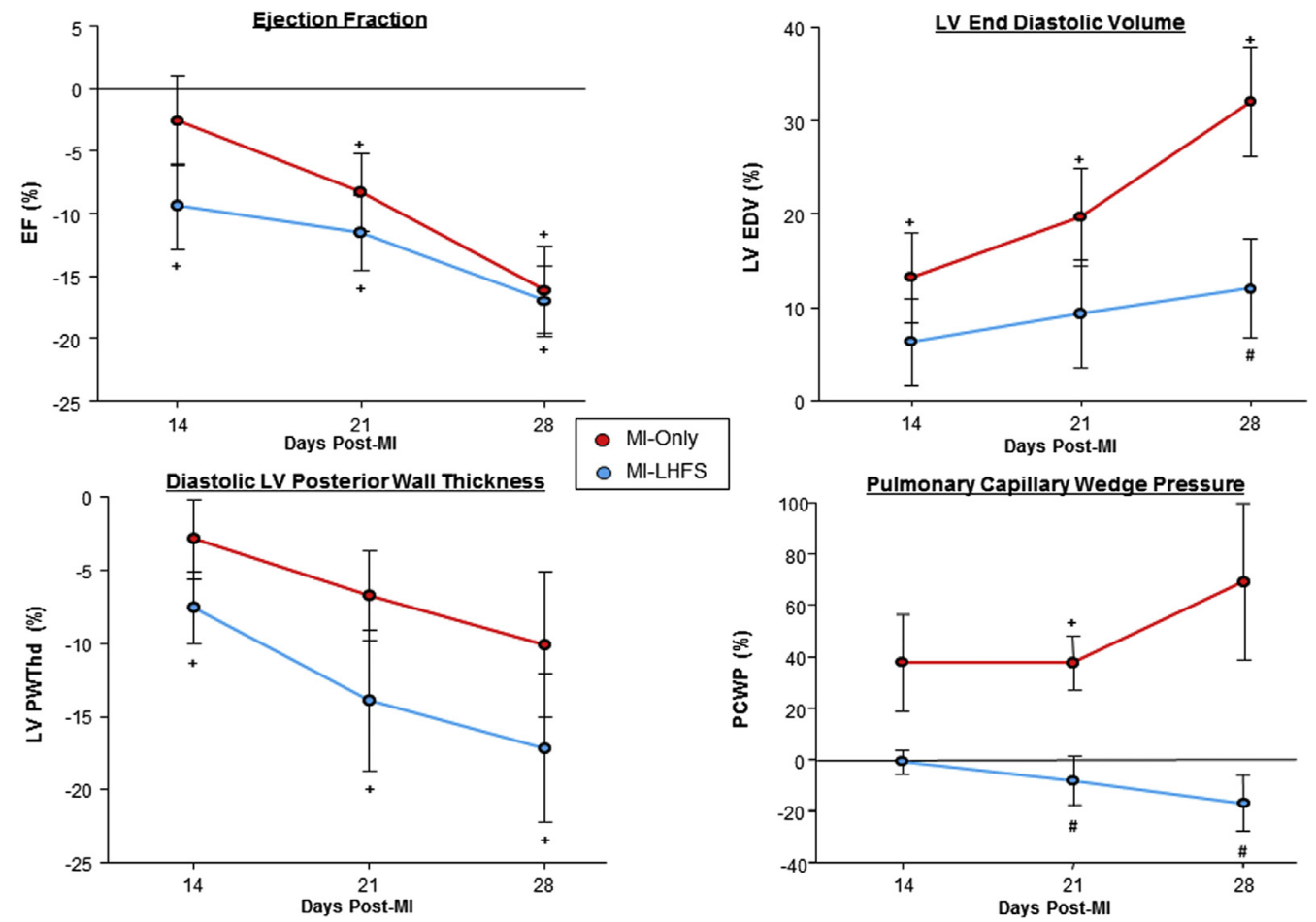

FIGURE 1. Changes in left ventricle $(L V)$ function and geometry as a function of 7-day post-myocardial infarction $(M I)$ values. Pigs were randomized to the localized high-frequency stimulation $(L H F S)$ protocol at MI induction, but the LHFS protocol was not initiated until after collection of the 7-day post-MI measurements. Thus, if LHFS influenced the post-MI remodeling process, then changes from this index value should have been realized. Top left, LV ejection fraction fell to a similar degree in both the MI only and LHFS group. Top right, LV end-diastolic volume increased as a function of time post-MI, but the relative rate of LV dilation was reduced in the LHFS group. Bottom left, LV posterior wall thickness, in this case at end-diastole, continued to fall at later postMI time points in the MI-only group but was attenuated in the LHFS group. Bottom right, Pulmonary capillary wedge pressure increased sharply in the MIonly groups at later post-MI time points but remained relatively unchanged in the LHFS group as a function of 7-day post-MI values. $+P<.05$ versus 7 -day post-MI values. $\# P<.05$ versus respective MI-only values. $E F$, Ejection fraction; $L V E D V$, left ventricular end diastolic volume; $P W T h d$, posterior wall thickness in diastole; $P C W P$, pulmonary capillary wedge pressure. 


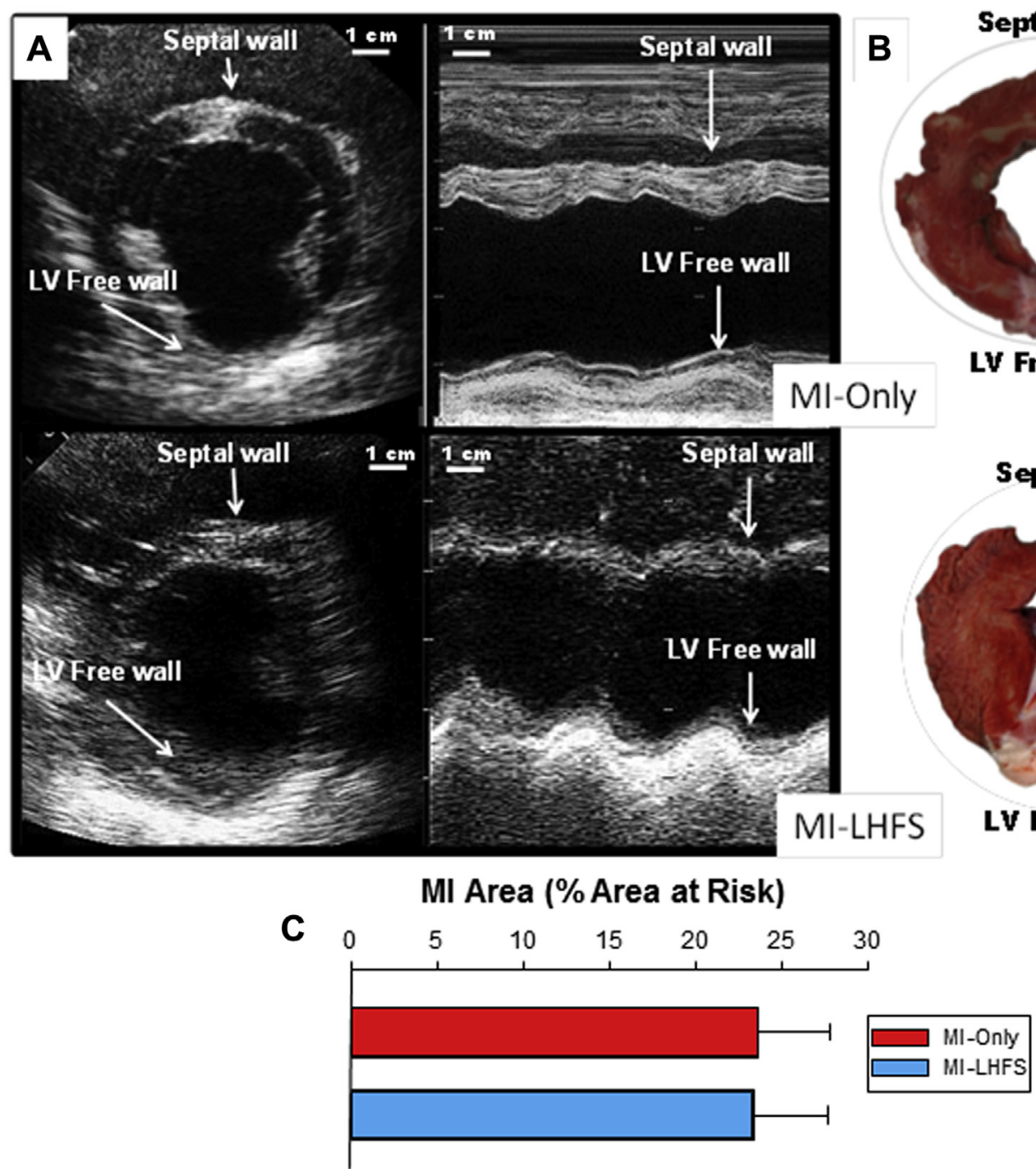

FIGURE 2. A, Representative transthoracic 2-dimensional targeted echocardiograms for a myocardial infarction ( $M I)$ only and localized high-frequency stimulation $(L H F S)$ study. The degree of left ventricle $(L V)$ wall thinning (LV free wall) at 28 days post-MI was reduced in the LHFS group. B, Circumferential LV sections taken at 28 days post-MI demonstrated increased MI thickness with LHFS. C, Although the degree of adverse post-MI remodeling was reduced with LHFS, institution of this protocol at 7 days post-MI did not modify absolute MI size.

of LV dilation, as reflected as a percent change in LV enddiastolic volume, is consistent with past reports using this MI model, and this relative change is not dissimilar to past clinical post-MI reports. ${ }^{1-3,16-18}$ PCWP increased in the MI-only group at longer post-MI time points but remained unchanged from 7-day values in the MI-LHFS group. LV ejection fraction fell in an equivalent direction and magnitude in both groups.

In terms of treatment and interactive effects, the multiway ANOVA revealed that time post-MI was a significant independent effect with respect to LV end-diastolic volume, wall thickness, ejection fraction, and PCWP (all $F$ values $>7 ; P<.05$ ). With respect to an independent treatment (ie, LHFS) effect, LV wall thickness was identified ( $F$ value $=5 ; P=.003)$. Finally, a significant time-treatment interaction was identified for $\mathrm{PCWP}(F$ value $=3.28 ; P=.03)$. This observation suggests that LHFS influenced this index of LV filling pressures in both a time and treatment-dependent fashion.

Representative LV cross-sectional views used for histochemical assessment of MI size are shown in Figure 2 along with concordant short-axis targeted LV echocardiographic views. MI wall thickness was notably greater in the LHFS group, which is concordant with the LV posterior wall thickness measurements taken by echocardiography. As a function of area at risk (complete circumflex perfusion region); however, absolute MI size was equivalent between groups. Thus, LHFS did not modify the degree of myocardial injury and therefore MI size but did alter both LV and MI regional geometry. 


\section{DISCUSSION}

Although significant advancements have been made in the timing and approach for coronary revascularization, and as a result significant improvements in survival with acute coronary syndromes have been realized, therapeutic strategies that target the residual region of myocardial injury/MI have not kept pace. The structure and the geometry of the MI region changes in a time-dependent manner characterized by MI thinning, increased stress-strain on the viable LV myocardium, eventually leading to LV dilation and failure-a process generically termed post-MI remodeling. The goal of the present study was to examine whether and to what degree institution of LHFS could modify the progression of post-MI remodeling in the context of a formed, pre-existing MI. Using conventional pacemaker leads and an implantable pacemaker modified to deliver high rate pacing, the current project instituted LHFS at 7 days post-MI and identified several unique findings. Firstly, the degree of LV wall thinning was reduced, as was the trajectory of LV dilation with LHFS. Secondly, the increase in PCWP that occurred during the post-MI period, which is a recognized hemodynamic index for the progression of LV failure, was abrogated with LHFS. Third, these changes in LV remodeling and function by LHFS were not due to an absolute reduction in MI size (hence not related to myocardial salvage or differences in initial MI injury), suggesting that LHFS interrupts the MI expansion process itself through local changes in the biophysical properties of the MI region. These new and unique findings provide the proof of concept that the preformed MI region is a modifiable structure and is responsive to electrical stimulation using translationally and clinically relevant approaches.

Past studies have demonstrated that fibroblasts contain ion channels and a resting membrane potential, suggestive that these cells would be responsive to electrical stimulation. ${ }^{22-24}$ Within the formed MI region, this cell type predominates and the remodeled extracellular matrix forms a natural electrical isolator from viable myocardium. We employed an electrical stimulation protocol within the formed MI region using specific stimulation parameters, whereby our initial electrical mapping studies determined that there was no propagation of the electrical stimulus from the targeted area when a pulse amplitude and duration of $0.8 \mathrm{~V}$ and $0.05 \mathrm{~ms}$ are used, respectively. ${ }^{11}$ Accordingly, we adopted these stimulation parameters for the current study in which LHFS was implemented at 7 days post-MI. Using this approach, beneficial effects on post-MI remodeling and the progression to LV failure was realized. However, it cannot be discounted that there may be additional permutations of the LHFS protocol that may yield optimal results but that was not the intent of the present study. Although LV wall thickness (hence MI thinning) and dilation were reduced with
LHFS, this did not translate into an identifiable improvement in LV ejection fraction. There are several considerations to be taken into account with this observation. It may be that the beneficial effects on LV ejection fraction, that is a slowing in the progression of this index of LV systolic function, would require a longer follow-up period. There is some evidence to suggest that this may be the case: key indices of adverse LV remodeling were being attenuated with later post-MI time points, and PCWP, another index of LV failure, was abrogated with LHFS. It should also be recognized that the most important predictor of long-term outcomes in patients post-MI is not LV ejection fraction but rather adverse LV remodeling, such as LV dilation, and are commonly measured as response variables in clinical studies. ${ }^{1-3,15-18,25-28}$ Thus, the findings from the present study regarding the use of LHFS at a relevant post-MI time point attenuated indices of LV remodeling holds clinical relevance.

Although the use of LHFS within the formed MI region itself is novel, the concept of localized myocardial stimulation post-MI has been described previously. ${ }^{15,28}$ These studies focused on myocardial stimulation of the viable border zone myocardium to achieve potentiation of contraction during systole. The concept of these past studies was that electrical stimulation of the border zone would yield favorable effects in terms of myocardial stiffening, alter local stress-strain patterns in the border zone-MI region, and in turn attenuate MI expansion and LV dilation. The Post-Myocardial Infarction Remodeling Prevention Therapy (PRomPT) trial was such a study, whereby patients with a significant and documented MI underwent local electrical stimulation within 10 days post-MI, but the primary end point, a reduction in LV dilation, was not achieved. ${ }^{15}$ It must be emphasized that there are significant and substantial differences between this past clinical study and the LHFS protocol. First, LHFS locally stimulates the MI region and does not propagate the electrical stimulus to viable myocardium. Secondly, LHFS is designed to alter the MI region by high-frequency low-amplitude stimulation and by the very definition of this protocol is not intended to cause acute changes in myocardial regional function or performance. Although there are fundamental, distinct differences between the PRomPT trial and this LHFS study, it is notable that this past clinical trial demonstrated the feasibility of introducing a stimulation protocol at an early postMI time point.

The preponderance of past preclinical and translational studies has focused upon mitigating the degree of myocardial injury and thus reducing the MI size that arises from the initial ischemic insult. The present study examined a different aspect of the MI process, whereby a therapeutic strategy was instituted within an established MI region. Specifically, LHFS was instituted at 7 days post-MI, 
whereby the initial wound healing and inflammatory response have subsided. This time point holds significance for those patients with a well-defined, preformed MI that is accessible either through a direct surgical or minimally invasive approach. For example, it has been identified that optimal timing for surgical coronary revascularization in the context of an MI is approximately 3 days following the initial event. ${ }^{13,14,29,30}$ The present study used epicardial pacing leads and programming modification of a commercially available pacemaker, both of which have been used clinically, and thus the translation of this preclinical study to the context of cardiac surgery and MI holds relevance. In the present study, the ligation of specific targeted coronary arteries was performed to create a uniform, transmural MI. As such, this resulted in an equivalent degree of myocardial injury and MI size and therefore reduced this potential confounding variable when examining the treatment effect of LHFS. The initiation of LHFS at 7 days post-MI and MI size evaluation at 28 days post-MI (21-day total treatment duration of LHFS) revealed no changes in absolute MI size. The purpose of this MI size measurement was to select a uniform region of the LV to assess the extent of myocardial injury, and this approach has been shown to reasonably approximate total MI size when compared with magnetic resonance imaging approaches. ${ }^{12}$ This observation, combined with the fact that LHFS reduced LV dilation and wall thinning, would suggest that the mechanism for LHFS is to attenuate MI expansion/remodeling rather than 1 of myocardial salvage or regeneration. Further evidence to support this postulate is that at post-MI day 7, before the institution of LHFS, absolute LV volumes were higher in those animals randomized to the active treatment group. The degree of LV dilation early post-MI has been identified as a harbinger of rapid LV remodeling, continued dilation, and pump dysfunction with longer post-MI intervals. ${ }^{1,16-18}$ Thus, the attenuation in LV dilation with the institution of LHFS at later post-MI time points would provide further support that this treatment approach altered local structure-function pathways and in turn the natural history of the post-MI remodeling process. Based on this observation, future studies by which extracellular matrix synthesis or degradation pathways are influenced by LHFS would be appropriate.

There are also study limitations that must be recognized, which include the MI model and the LHFS protocol. The coronary ligation model may not fully recapitulate the MI, which is caused by ischemia-reperfusion, and whether and to what degree an MI at different geometric locations would be responsive to LHFS remains to be determined. This was a proof of principle study and it must be recognized that the sample sizes were small and assumed that the relative reduction in LV dilation with LHFS would be significant and associated with a low degree of variation. Although these initial criteria were established a priori, the intrinsic variability (standard deviation) with respect to the percent change in LV end-diastolic volume from day 7 post-MI values was higher than projected. As such, the statistical power may indeed be reduced to nearly $50 \%$ of initial study estimates. With this reduced statistical power, potential treatment and time interactive effects, and independent treatment effects may have not been identified. Nevertheless, a significant treatment effect of LHFS was observed with respect to LV remodeling and an important time-treatment interaction was observed for a key determinant of heart failure progression (ie, PCWP). The LHFS stimulation protocol was based on initial preliminary studies, ${ }^{11}$ and whether continuous stimulation or some alternating pattern of electrical stimulation of the MI region would achieve similar results remains unclear. However, it must be recognized that the permutations on frequency, amplitude, and duration would yield problematic issues for large animal post-MI studies. Despite these considerations, the present study provides a clear proof of principle that LHFS can favorably affect the post-MI remodeling process in the context of an existing, preformed MI.

\section{CONCLUSIONS}

LHFS represents a novel concept in that electrical stimulation may be used to attenuate indices of adverse LV remodeling and potentially prevent the progression of LV failure secondary to MI. Specifically, the reduction in LV dilation (end-diastolic volume), which was achieved by LHFS, has been identified as a critical and often primary response variable when evaluating clinical progression of heart failure. ${ }^{1,15,25-27}$ More importantly, LHFS may be complementary to pharmacologic/cell-based therapies under development for early post-MI treatment. Although significant efforts are underway to improve myocardial salvage or induce regeneration post-MI, the LHFS approach presents a simple and novel paradigm to change what has been considered a refractory tissue - the formed, maturing MI region. Although additional studies are necessary, 1 future direction would be to consider placement of a stimulation electrode in a preformed, existing MI region in patients undergoing coronary revascularization. As such, LHFS may provide a complementary/additive therapy by transforming the MI region in addition to improving myocardial blood flow.

\section{Conflict of Interest Statement}

Dr Ruble is an employee Boston Scientific Inc, a company that manufactures a wide range of devices, including those for including cardiovascular disease. However, Dr Ruble played no role in the study analysis and interpretation. The pacemaker leads, pacemakers, and programmer were funded through an investigator-sponsored study agreement with Boston Scientific. All other authors have nothing to disclose with regard to commercial support. 


\section{References}

1. St John Sutton M, Pfeffer MA, Moye L, Plappert T, Rouleau JL, Lamas G, et al. Cardiovascular death and left ventricular remodeling two years after myocardial infarction: baseline predictors and impact of long-term use of captopril: information from the survival and ventricular enlargement (SAVE) trial. Circulation. 1997;96:3294-9.

2. Webb CS, Bonnema DD, Ahmed SH, Leonardi AH, McClure CD, Clark LL, et al. Specific temporal profile of matrix metalloproteinase release occurs in patients after myocardial infarction: relation to left ventricular remodeling. Circulation. 2006;114:1020-7.

3. Squire IB, Evans J, Ng LL, Loftus IM, Thompson MM. Plasma MMP-9 and MMP-2 following acute myocardial infarction in man: correlation with echocardiographic and neurohumoral parameters of left ventricular dysfunction. J Card Fail. 2004;10:328-33.

4. Cleutjens JP, Creemers EE. Integration of concepts: cardiac extracellular matrix remodeling after myocardial infarction. J Card Fail. 2002;8(6 Suppl): S344-8.

5. Lindsey ML, Escobar GP, Mukherjee R, Goshorn DK, Sheats NJ, Bruce JA, et al. Matrix metalloproteinase- 7 affects connexin-43 levels, electrical conduction, and survival after myocardial infarction. Circulation. 2006;113:2919-28.

6. Mukherjee R, Brinsa TA, Dowdy KB, Scott AA, Baskin JM, Deschamps AM, et al. Myocardial infarct expansion and matrix metalloproteinase inhibition. Circulation. 2003;107:618-25.

7. Spinale FG. Myocardial matrix remodeling and the matrix metalloproteinases: influence on cardiac form and function. Physiol Rev. 2007;87:1285-342.

8. Lindsey ML, Iyer RP, Jung M, DeLeon-Pennell KY, Ma Y. Matrix metalloproteinases as input and output signals for post-myocardial infarction remodeling. J Mol Cell Cardiol. 2016;91:134-40.

9. D'Elia N, D'hooge J, Marwick TH. Association between myocardial mechanics and ischemic LV remodeling. JACC Cardiovasc Imag. 2015;8:1430-43.

10. Carrick D, Haig C, Rauhalammi S, Ahmed N, Mordi I, McEntegart M, et al. Pathophysiology of LV remodeling in survivors of STEMI: Inflammation, remote myocardium, and prognosis. JACC Cardiovasc Imag. 2015;8:779-89.

11. Mukherjee R, Rivers WT, Ruddy JM, Matthews RG, Koval CN, Plyler RA, et al. Long-term localized high-frequency electric stimulation within the myocardial infarct: effects on matrix metalloproteinases and regional remodeling. Circulation. 2010;122:20-32

12. Eckhouse SR, Purcell BP, Oelsen JM, Logdon CB, Rawls WF, Patel RK, et al. Local hydrogel release of recombinant TIMP-3 attenuates adverse left ventricular remodeling after experimental myocardial infarction. Sci Transl Med. 2014;6: $223 \mathrm{ra} 21$.

13. Parikh SV, de Lemos JA, Jessen ME, Brilakis ES, Ohman EM, Chen AY, et al Timing of in-hospital coronary artery bypass graft surgery for non-ST-segment elevation myocardial infarction patients results from the national cardiovascular data registry ACTION registry-GWTG (acute coronary treatment and intervention outcomes network registry-get with the guidelines). JACC Cardiovasc Interv. 2010;3:419-27.

14. Davierwala PM, Verevkin A, Leontyev S, Misfeld M, Borger MA, Mohr FW. Does timing of coronary artery bypass surgery affect early and long-term outcomes in patients with non-st-segment-elevation myocardial infarction? Circulation. 2015;132:731-40.

15. Stone GW, Chung ES, Stancak B, Svendsen JH, Fischer TM, Kueffer F, et al. Peri-infarct zone pacing to prevent adverse left ventricular remodelling in patients with large myocardial infarction. Eur Heart J. 2016;37:484-93.
16. Iliceto S, Scrutinio D, Bruzzi P, D’Ambrosio G, Boni L, Di Biase M, et al. Effects of L-carnitine administration on left ventricular remodeling after acute anterior myocardial infarction: the L-carnitine ecocardiografia digitalizzata infarto miocardico (CEDIM) trial. J Am Coll Cardiol. 1995;26:380-7.

17. White HD, Norris RM, Brown MA, Brandt PW, Whitlock RM, Wild CJ. Left ventricular end-systolic volume as the major determinant of survival after recovery from myocardial infarction. Circulation. 1987;76:44-51.

18. Pfeffer MA, Braunwald E, Moyé LA, Basta L, Brown EJ Jr, Cuddy TE, et al. Effect of captopril on mortality and morbidity in patients with left ventricular dysfunction after myocardial infarction. Results of the survival and ventricular enlargement trial. The SAVE Investigators. N Engl J Med. 1992;327:669-77.

19. National Institutes of Health. Guide for the Care and Use of Laboratory Animals. 8th ed. Washington: National Academies Press; 2011.

20. Ommen SR, Nishimura RA, Appleton CP, Miller FA, Oh JK, Redfield MM, et al. Clinical utility of Doppler echocardiography and tissue Doppler imaging in the estimation of left ventricular filling pressures: a comparative simultaneous Doppler-catheterization study. Circulation. 2000;102:1788-94.

21. Nagueh SF, Middleton KJ, Kopelen HA, Zoghbi WA, Quiñones MA. Doppler tissue imaging: a noninvasive technique for evaluation of left ventricular relaxation and estimation of filling pressures. J Am Coll Cardiol. 1997;30:1527-33.

22. He ML, Liu WJ, Sun HY, Wu W, Liu J, Tse HF, et al. Effects of ion channels on proliferation in cultured human cardiac fibroblasts. J Mol Cell Cardiol. 2011;51: 198-206.

23. Shibukawa Y, Chilton EL, Maccannell KA, Clark RB, Giles WR. K+ current activated by depolarization in cardiac fibroblasts. Biophys J. 2005;88:3924-35.

24. Poulet C, Künzel S, Büttner E, Lindner D, Westermann D, Ravens U. Altered physiological functions and ion currents in atrial fibroblasts from patients with chronic atrial fibrillation. Physiol Rep. 2016;4:e12681.

25. Zannad F, De Ferrari GM, Tuinenburg AE, Wright D, Brugada J, Butter C, et al Chronic vagal stimulation for the treatment of low ejection fraction heart failure: results of the NEural Cardiac TherApy foR heart failure (NECTAR-HF) randomized controlled trial. Eur Heart J. 2015;36:425-33.

26. Weiner RB, Baggish AL, Chen-Tournoux A, Marshall JE, Gaggin HK Bhardwaj A, et al. Improvement in structural and functional echocardiographic parameters during chronic heart failure therapy guided by natriuretic peptides: mechanistic insights from the ProBNP outpatient tailored chronic heart failure (PROTECT) study. Eur J Heart Fail. 2013;15:342-51.

27. Weir RA, Mark PB, Petrie CJ, Clements S, Steedman T, Ford I, et al. Left ventricular remodeling after acute myocardial infarction: does eplerenone have an effect? Am Heart J. 2009;157:1088-96.

28. Chung ES, Dan D, Solomon SD, Bank AJ, Pastore J, Iyer A, et al. Effect of periinfarct pacing early after myocardial infarction: results of the prevention of myocardial enlargement and dilatation post myocardial infarction study. Circ Heart Fail. 2010;3:650-8.

29. Lee DC, Oz MC, Weinberg AD, Ting W. Appropriate timing of surgical intervention after transmural acute myocardial infarction. J Thorac Cardiovasc Surg. 2003;125:115-9; discussion 119-0.

30. Lee DC, Oz MC, Weinberg AD, Lin SX, Ting W. Optimal timing of revascularization: transmural versus nontransmural acute myocardial infarction. Ann Thorac Surg. 2001;71:1197-202.

Key Words: electrical stimulation, myocardial infarction, ventricular geometry 